\title{
Análisis didáctico de las intervenciones de los docentes en los foros de discusión en cursos de postgrado en modalidad a distancia
}

\author{
Wellington Mazzotti*
}

"Todos los hombres necesitan comunicarse... El hombre requiere salir de sí mismo para contrarrestar sus limitaciones, para ir completando su ser. Busca transferir su pensamiento, su intimidad para proyectarse fuera de sí...."1

\section{Resumen}

Este artículo refiere a un estudio sobre la participación de docentes y alumnos en foros de discusión en cursos de postgrado realizados en modalidad a distancia. Se analizaron las intervenciones de los docentes y alumnos en dos aspectos: a) cantidad y frecuencia de las intervenciones de los docentes y el impacto que éstas produjeron en la dinámica comunicacional del grupo virtual y b) modos de intervención de los docentes en los foros.

De las observaciones realizadas se puede establecer que existe una relación directa entre la propuesta de intervención del docente que dirige el foro y el patrón comunicacional del grupo virtual. Un docente que despliega un abanico de estrategias de intervención y una activa participación en el foro provoca una gran interactividad entre los participantes del grupo virtual. Por el contrario, una pobre intervención en calidad y cantidad por parte del docente produce poca participación de los alumnos con aportes de escasa significación.

Se describen aquí los modos de intervención, técnicas y estrategias utilizadas por los docentes para animar los foros de discusión.

\begin{abstract}
This article describes research about the participation of tutors and students in discussions forums, in e-learning postgraduate courses. Two aspects of the interventions were analyzed: a) Quantity and frequency of tutor's interventions and their impact in the communicational dynamics of the virtual group;

b) Modes of intervention of tutors in discussions forums.
\end{abstract}

The research findings established that there is a direct relationship between the tutor's purpose of intervention and the communicational pattern of the virtual group. A tutor displaying a variety of intervention strategies and an active participation in the forum achieves a high degree of interactivity between the students. On the other hand, tutor interventions that are poor in quality and quantity yield low participation of students and poor contributions. This article describes the intervention modes, techniques and strategies used by tutors in order to animate the forums. 


\section{I ntroducción}

El proceso del aprendizaje del ser humano es un fenómeno complejo. En él intervienen mecanismos que permiten al individuo aprender en estrecha relación entre el ser y el objeto de conocimiento. Pero para que esta relación se vea potenciada es preciso que el sujeto interactúe con su entorno para desarrolla funciones psíquicas superiores tales como la internalización. El aprendizaje de una persona está mediado por la cultura, el sujeto ante todo es un ser social, no sólo es activo en su proceso de aprendizaje sino, ante todo, interactivo.

Cuando los procesos de enseñanza y de aprendizaje tienen lugar en modalidad a distancia, deben instrumentarse mecanismos que permitan a los alumnos estar en contacto con los saberes y potenciar los intercambios interpersonales. Por esto las herramientas de comunicación -foro de discusión, conversaciones sincrónicas (chat), teleconferencias, correo electrónicoadquieren una importancia vital para provocar los aprendizajes que tienen lugar en estos entornos no presenciales.

Los foros constituyen una de las estrategias interactivas más aptas para propiciar estos intercambios virtuales interpersonales pues generan un espacio de colaboración y discusión en donde fermentan los procesos de aprendizaje. También se les denomina, muy apropiadamente, foros de discusión en Internet, grupos de diálogo o conferencias electrónicas (Icaza, 2000). En ellos, gracias a la interactividad y el control de la comunicación apoyados por recursos tecnológicos de emisor y receptor, docentes y alumnos utilizan esta plataforma informática para alojar información de interés (opiniones, ideas, soluciones, inconvenientes...) para que otros accedan a ella con la intención de preguntar, matizar, desarrollar o ponerla en entredicho públicamente. De esta forma se propicia la interacción con otras personas que tienen intereses comunes y se contribuye a ofrecer diversidad de criterios acerca de un mismo tópico.

Pero estos foros no sólo constituyen un espacio de debate, sino que también son una fuente de enriquecimiento del conocimiento para el docente, de información y entendimiento del comportamiento de los grupos virtuales y de los aprendizajes que tienen lugar en estos escenarios. Son por ello un objeto genuino y muy apto para la investigación educativa, entendiéndola como un conjunto de procedimientos para obtener conocimiento. ${ }^{1}$ Los foros, por participar en un proceso educativo, constituyen una realidad muy rica y compleja que puede dar lugar a miradas muy diversas, todas ellas interesantes porque aportan elementos para su comprensión.

Este articulo refiere al conocimiento emanado del trabajo de investigación realizado en los foros de discusión de los cursos a distancia correspondientes a los postgrados del Instituto de Educación de la Universidad ORT en el marco del trabajo de tesina de la Maestría en Educación Abierta y a Distancia realizada en la UNED. La investigación se centró en el estudio de los modos de intervención de los docentes en los foros de discusión y cómo éstos impactan en la dinámica del grupo virtual, en el entendido de que las intervenciones de los docentes deben fomentar una "interacción de calidad"2 , en el sentido que una verdadera interactividad es aquella que estimula el pensamiento, en niveles de análisis, síntesis, evaluación, como más elevados e interesantes. 


\section{Sobre los foros de discusión}

\subsection{Los foros de discusión}

Los grupos de diálogo o foros de discusión son comunidades virtuales ${ }^{3}$ en donde participan personas con un interés común que establecen una conversación electrónica a través de un soporte telemático sin necesidad de compartir un espacio físico común ni coincidir en el tiempo. En este espacio virtual se comparten ideas, reflexiones, aplicaciones de conocimiento, se hacen análisis de la temática plateada, dentro de un esquema de aprendizaje colaborativo en el que interviene un grupo de personas con perfiles académicos y profesionales, con expectativas, y objetivos comunes, dentro del cual intercambian mensajes, los que son publicados en una sección o página web. "Esta aplicación respeta el orden de las intervenciones y las enlaza conforme fueron generadas, con lo que mantiene en pantalla y al alcance de cualquier persona del grupo que se acerque al foro no sólo la relación completa de intervenciones, sino su estructura dinámica, es decir, qué propuso u opinó alguien y qué reacción y secuencia de intervenciones produjo en los demás" (García Aretio, 2001). La denominación de foro a esta herramienta informática evoca las grandes asambleas romanas en las que todos los integrantes del auditorio tenían oportunidad de debatir el tema puesto en consideración.

Existen distintos tipos de foro de discusión, pueden ser abiertos o cerrados, o sea de acceso libre para cualquier persona o restringido para un grupo de personas seleccionado según determinado criterio. Existen los foros moderados, no moderados y premoderados, dependiendo de cómo es la participación del moderador, si es que lo hay. Este estudio refiere a foros de discusión cerrados porque en éstos sólo pueden participar los alumnos pertenecientes a cursos de postgrado del Instituto de Educación de la Universidad ORT. Son moderados por el profesor de la materia.

\subsection{La tarea docente en el foro de discusión}

"Las modalidades comunicativas que se establecen en un foro permiten el desarrollo de espacios de relación social que promueven el aprendizaje interactivo" (Gallego, 2003). El docente debe procurar que esta conversación sea de calidad, no un simple intercambio de mensajes, sino que se logre una cierta profundidad en las reflexiones que conduzcan a procesos de aprendizaje. Por otro lado, es necesario promover una comunicación multidireccional para establecer un entrelazado social lo más amplio posible entre todos los integrantes del grupo, evitando la tendencia natural que lleva a reducir la comunicación al diálogo bidireccional exclusivo entre alumno y docente. Las modernas Tecnologías de la Información y la Comunicación ${ }^{4}$ permiten desarrollar una comunicación multidireccional con la cual se puede producir un verdadero diálogo gracias a la interacción entre el profesor y los alumnos, en forma individual o colectiva, y entre alumnos. Aquí, diálogo incluye la idea de que los humanos que se comunican están comprometidos en la construcción y cambio de significados y no simplemente en la transmisión de mensajes (Gallego et al, 2000).

La enseñanza en modalidad a distancia ${ }^{5}$ tiene una singularidad que hay que atender, los conocimientos se construyen a través del "diálogo mediado", a través de los "recursos mediadores", generando capacidad formativa y aprendizaje autónomo-participativo en las que el aprendiz interioriza el saber y reelabora el sentido de los mensajes recibidos (Medina, 2000).

El docente que participa en el foro de discusión tendrá una dificultad adicional que deberá sortear con astucia: detectar a través del medio telemático los diferentes estilos de aprendizaje ${ }^{6}$ que tienen los alumnos adultos que integran esta comunidad virtual. 
No cabe duda de que describir el comportamiento del grupo virtual, los estilos de aprendizaje individuales y los modos de intervención del docente y cómo estos inciden sobre las conductas y los aprendizajes de los participantes en el foro, nos permitirá reflexionar sobre las estrategias didácticas empleadas de modo que estas intervenciones pedagógicas permitan alcanzar los objetivos educativos propuestos. Si la estrategia de interacción fue adecuadamente diseñada, lo más seguro es que logre llevar a buen fin la comunicación buscada y, por ende, la tarea asignada. Pero las estrategias diseñadas para incentivar un proceso de aprendizaje no siempre son las mismas, la comunicación no se establece en las mismas condiciones por lo que el diseño de la intervención docente tiene que adaptarse a cada grupo. Existe, en algunos ámbitos educativos, la idea reduccionista de creer que la educación a distancia consiste básicamente en publicar buenos materiales en Internet. Éstos son muy necesarios, pero a un mismo nivel de importancia se encuentran las herramientas de comunicación en general y los foros de diálogo en particular, pues ellos son los que favorecen el aprendizaje colaborativo, que tiene sus fundamentos en la concepción del aprendizaje social de Vigotsky, asociado a la teoría social constructivista que supone establecer estrategias de enseñanza y de evaluación para propiciar en los estudiantes el desarrollo de un aprendizaje consciente y significativo en interacción con sus comunes (Bruno, 1999, citado por Brito, 2004).

\subsection{Las estrategias de enseñanzas en un nuevo escenario}

El nuevo modelo de enseñanza basado en las TIC demanda "al profesional de la educación que tenga otra concepción de su trabajo, que le exige más conocimiento, más tecnificación, más práctica reflexiva, más crítica, más trabajo cooperativo, más interrelación, más profesionalidad" (Castillejo, 1987) ${ }^{7}$. Las acciones del docente deberán responder a un nuevo modelo de planificación, integrando en forma ordenada, coherente, sistemática y secuencial, cada una de las herramientas de comunicación a utilizar (García Aretio, L. Unidad Didáctica 50: 72).

\subsection{Aspectos a tener en cuenta en los foros de discusión}

Una de las preguntas centrales que cabe formularse en el marco de los intercambios en los foros electrónicos es si las intervenciones de los docentes promueven los aprendizajes significativos $^{8}$ en los estudiantes, qué estrategias de enseñanza aplica el docente y si éstas están en consonancia con las características particulares de los estudiantes. En pocas palabras, conocer si el docente propicia la construcción del conocimiento. El enfoque constructivista considera que el alumno va construyendo su propia representación interna del conocimiento a través de la interacción con los materiales, el asesor y el medio.

Por otra parte, el hecho de estudiar a distancia, puede representar para algunos estudiantes un sentimiento de soledad, que hay que contrarrestar de alguna manera. Esta cuestión no es sencilla, si se considera que los medios tecnológicos pueden resultar en primera instancia fríos. El sentido de la distancia asumido por los estudiantes sobre la Institución, dependerá principalmente de la rapidez y la calidad de las respuestas que reciben a sus requerimientos. Si bien la incorporación de las TIC facilita las oportunidades de contacto con el especialista, el tutor o el moderador, de poco sirven y su potencial disminuye si el estudiante no sabe cómo, con qué o a partir de qué modo establecer el vínculo con ellas. Cuando la población estudiantil es adulta, los profesores tienen que tener en cuenta los aportes de la androgogía ${ }^{9}$ ya que existen diferencias significativas entre la forma de aprender del adulto respecto a la de los niños y jóvenes. El docente, profesional de la educación, además de dominar el campo disciplinar, debe tener una capacidad para el autodesarrollo profesional mediante un sistemático autoanálisis ${ }^{10}$ de su labor, siendo la investigación en el aula el sitio adecuado para la educación presencial y los foros de discusión en la educación a distancia. 


\subsection{Factores intrínsecos que condicionan la interrelación personal}

La interrelación personal mediada por el ordenador está condicionada por factores que la favorecen o la obstaculizan. Conocerlos es importante para quien debe moderar los foros. Estos factores pueden ser intrínsecos o extrínsecos a los sujetos. Los factores intrínsecos son aquellos que inciden en la comunicación y que están en estrecha vinculación con la conducta que adopta la persona que aprende. El nuevo escenario en donde tienen lugar los procesos de enseñanza y de aprendizaje suponen un cambio cultural que provoca distintas reacciones en las diferentes personas. Para los adultos de hoy, este cambio implica nuevas formas de interrelación y de intercambio. Las conductas de interacción en educación a distancia no son las mismas que las que se dan en un aula tradicional. El cambio de entorno implica una adaptación del sujeto a una nueva manera de relacionarse con el exterior y pesan distintos factores como ser la relación con la tecnología, las características de la personalidad vinculadas con el desenvolvimiento en los grupos presenciales y la historia personal de cada uno en función de distintas experiencias vividas en aulas presenciales, en donde los aprendizajes se dieron bajo la presencia física de otras personas, con una comunicación regida por su triple vertiente (verbal, no verbal y paraverbal).

La decodificación de la interpretación y la mala interpretación en la comunicación por computadora han sido objeto de estudio de numerosos trabajos. La reducción de la comunicación al texto escrito, sin la presencia de otros elementos, como el tono, la postura corporal y otros gestos que acompañan a la palabra limitan notablemente la riqueza de la interacción y exigen una adaptación a la nueva forma de comunicación.

En el estudio realizado en los años 1999-2000 sobre los factores intrínsecos que inciden en las interacciones interpersonales que tienen lugar en escenarios educativos no presenciales basados en inter/intranet en adultos (Mazzotti y Laurta) surgieron como los más relevantes los siguientes:

\begin{tabular}{|l|l|}
\hline \multicolumn{2}{|c|}{ Factores intrínsecos } \\
\hline Favorecen las interacciones & Obstaculizan las interacciones \\
\hline $\begin{array}{l}\text { Percepción de que el curso a distancia es } \\
\text { más personalizado. }\end{array}$ & $\begin{array}{l}\text { Ausencia de la comunicación no verbal } \\
\text { o paraverbal. }\end{array}$ \\
\hline $\begin{array}{l}\text { Facilitador de la comunicación a las personas } \\
\text { que son tímidas o de baja autoestima. }\end{array}$ & $\begin{array}{l}\text { Ausencia del conocimiento personal del } \\
\text { otro sujeto. }\end{array}$ \\
\hline Percepción de no sentirse aislado. & $\begin{array}{l}\text { Falta de conciencia grupal (la interacción } \\
\text { tiende a realizarse sólo con el docente/tutor). }\end{array}$ \\
\hline $\begin{array}{l}\text { Anulación de las inhibiciones que se generan } \\
\text { en los grupos presenciales en los que } \\
\text { participan colegas o superiores jerárquicos. }\end{array}$ & $\begin{array}{l}\text { Percepción de reducir las interacciones a } \\
\text { consulta de dudas. }\end{array}$ \\
\hline $\begin{array}{l}\text { Historia personal de buenas experiencias } \\
\text { educativas (formación académica formal } \\
\text { o no formal). }\end{array}$ & $\begin{array}{l}\text { Características personales (falta de constancia } \\
\text { para trabajar frente a un PC, dificultad para la } \\
\text { expresión escrita). }\end{array}$ \\
\hline $\begin{array}{l}\text { Conocimientos y habilidades en el área de la } \\
\text { informática (usuario competente). }\end{array}$ & Percepción del curso como de autoformación. \\
\hline & $\begin{array}{l}\text { Dificultad en el manejo de la ansiedad en el } \\
\text { intercambio asincrónico. }\end{array}$ \\
\hline
\end{tabular}




\subsection{Factores extrínsecos que condicionan la interrelación personal}

Existen además otros factores externos a los sujetos que favorecen u obstaculizan la interrelación personal en un proceso de aprendizaje a distancia, denominados factores extrínsecos. Éstos condicionan la comunicación en este nuevo entorno ya que implica un sistema tecnificado que modifica el acceso mismo al proceso de aprendizaje. Las nuevas tecnologías de la información y la comunicación permiten crear nuevos entornos comunicacionales que son muy propicios para la enseñanza a distancia porque no sólo permiten el acceso a una cantidad de materiales multimediáticos de alta calidad sino "sobre todo porque permiten la interacción, y en algunos casos la interacción en tiempo real, de todos los usuarios del sistema" (Cabero). Pero para que esto tenga lugar es necesario asegurar que exista un real acceso a la red de comunicaciones. No sólo que el acceso esté permitido sino que además se pueda conectar a la red fácilmente, sin trámites, sin necesidad de solicitar autorización y en el momentoen el que el estudiante lo desee. Otros factores como la sobrecarga de las líneas o los costos por conexión no pueden generar preocupación o mayores dificultades a los participantes de los cursos (Madon, 1995: 208). Otros autores señalan como una clave importante para el éxito de los aprendizajes en la educación a distancia, "el mantenimiento de una interacción consistente y de calidad" (McGiven, 1994) ${ }^{11}$.

En el mismo estudio mencionado anteriormente, se detectaron los siguientes factores extrínsecos (Mazzotti y Laurta, 2000):

\begin{tabular}{|l|l|}
\hline \multicolumn{2}{|c|}{ Factores extrínsecos } \\
\hline Favorecen las interacciones. & Obstaculizan las interacciones. \\
\hline $\begin{array}{l}\text { Tener en línea un profesor para interactuar } \\
\text { sincrónicamente. }\end{array}$ & $\begin{array}{l}\text { Falta de experiencia de los alumnos y los } \\
\text { docentes en curso a distancia. }\end{array}$ \\
\hline Tener una base de datos de consulta. & $\begin{array}{l}\text { Falta de un ámbito físico dedicado } \\
\text { al aprendizaje. }\end{array}$ \\
\hline $\begin{array}{l}\text { Poseer referencias concretas de los otros } \\
\text { participantes (fotos e instancias presenciales). }\end{array}$ & $\begin{array}{l}\text { Falta de acceso real al curso a distancia } \\
\text { (disponibilidad de tiempo, de equipo, de } \\
\text { permisos de acceso y de autorización de su } \\
\text { superior). }\end{array}$ \\
\hline
\end{tabular}

\section{Análisis didáctico de las intervenciones de los docentes en foros de discusión en cursos de postgrados en modalidad a distancia}

Este estudio tiene el propósito de hacer una mirada reflexiva sobre los foros de discusión a fin de poner de manifiesto las características de la intervención de los docentes. Mediante la creación de una serie de indicadores se obtiene información que permite al docente conocer el patrón de comunicación del grupo. Por otro lado, a través de una categorización de las intervenciones del docente se puede analizar si sus estrategias didácticas ${ }^{12}$ fueron las más ajustadas a las características del grupo en cuestión. No se pretende en este estudio profundizar en las formas de gestión de los foros por parte de los docentes. Éste es un tema muy relevante pero sería objeto de otra investigación. 
Subyace en el centro de esta investigación la idea de que los foros de discusión son espacios que los formadores a distancia disponen para promover el sentido de pertenencia a un grupo o a una clase. La actividad del foro va a depender en gran medida de la capacidad de los tutores para responder e incentiva la participación de los alumnos. La telegestión obliga a establecer procedimientos para que los tutores de un curso gestionen las intervenciones de los alumnos (C. Marcelo et al).

El escenario en donde transcurre esta investigación son los foros de discusión de las asignaturas 1 y 2 que se desarrollaron en modalidad a distancia, con las generaciones 2000 y 2001 del Diploma en Educación, curso de Postgrado del Instituto de Educación de la Universidad ORT. Se trata de foros cerrados puesto que en ellos sólo pueden participar los alumnos inscriptos en los cursos. Los participantes son docentes uruguayos, maestros o profesores en actividad, que realizan estudios de postgrado. El grupo que participó en el foro del año 2000 estuvo compuesto por 31 alumnos mientras que el grupo que participó en el foro del año 2001 estuvo integrado por 18 alumnos.

Se analizaron las intervenciones de los docentes y alumnos en dos aspectos:

a) cantidad y frecuencia de las intervenciones de los docentes y el impacto que produjeron en la dinámica comunicacional del grupo virtual.

b) modos de intervención de los docentes en los foros.

Para el estudio del primer aspecto se definió una serie de indicadores, entre otros, la cantidad de intervenciones totales en el foro, la cantidad de intervenciones de los alumnos, la cantidad de intervenciones de los docentes, el promedio de intervenciones, el porcentaje de alumnos que participan en forma activa en el foro, la cantidad de intervenciones directas entre los alumnos, el promedio de intervención del docente en el foro por día, y el tiempo de demora de respuesta del docente ante requerimientos de los alumnos. Además se determinó en qué días y en qué horarios los alumnos intervienen con mayor frecuencia ${ }^{13}$.

\section{Categorización de las intervenciones didácticas de los docentes en foros de discusión analizados}

Luego de realizar el análisis de texto de los cuatro foros de discusión correspondientes a las asignaturas 1 y 2 de los años 2000 y 2001, se llevó a cabo una categorización de las intervenciones de los docentes. A continuación se describen las categorías construidas, dando algunos ejemplos a modo de ilustración:

\section{Intervenciones para:}

1. Iniciar una línea temática de discusión

Una de las técnicas que emplean los docentes para revitalizar las discusiones en los foros es la de iniciar líneas temáticas una vez que la conversación del tema está por agotarse. El docente a cargo del foro planifica, antes de iniciarlo, cuáles son los ejes temáticos que a su juicio se deben abordar a lo largo del foro. Luego los administra en función de cómo se va desarrollando el dialogado.

Ejemplo:

"Estimados colegas, hemos recibido con agrado los primeros intentos de comunicación e intercambio por parte de ustedes. Es por ello que quiero invitarlos a iniciar una reflexión colectiva en torno a las temáticas que nos convocan..." 


\section{Ejemplo:}

"Didáctica: entre querer enseñar y saber enseñar.

Hola nuevamente: ahora comenzamos un nuevo foro, dentro de este seminario (Concepciones de la enseñanza). Vieron qué lindo nombre le di a este foro "Didáctica: entre querer enseñar y saber enseñar" (es el título de un libro sobre didáctica que estoy escribiendo. Espero tenerlo pronto para setiembre de este año).

Bien. Vayamos al título. Quisiera detenerme en dos aspectos: la intencionalidad de la..."

\section{Encauzar una discusión}

Otro de los modos de intervención observada en los docentes es el de encauzar la discusión cuando ésta se va desviando del eje central. Con mucho respeto hacia la participación de los alumnos, se propone dar una nueva mirada al tema en el sentido que al docente le parece conveniente.

Ejemplo:

"Muy valiosos los aportes de María ${ }^{14}$. Creo que estamos avanzando en nuestro análisis conjunto. Simplemente para confirmar alguna duda que nos dejaste planteada, les aclaro que fue efectivamente en 1973 en que se aprobó la llamada "Ley de Educación" (Nro. 14.101), que ....".

Ejemplo:

"Estimados colegas: volvemos a la ruta, continuamos recorriendo el camino de la Didáctica (este viaje ha tenido muchas paradas y desvíos y eso ha sido muy bueno). Sus intervenciones y contribuciones vienen siendo muy importantes. No duden en seguir en esta línea de construcción colectiva. Esas paradas y desvíos a los que hacía referencia son ejemplos notables de emergentes, algunos dentro de lo programático (es decir, dentro de lo que está previsto en el seminario), pero otros constituyen emergentes fuera de lo programático. Todos ellos cooperan en la revisión y ..."

\section{Generar participación de los alumnos en el foro}

Una de las tareas principales del docente que está a cargo del foro es la de motivar a los alumnos para que participen en él. Una práctica habitual de los docentes es la de finalizar sus intervenciones requiriendo participación de los alumnos: se les solicita su opinión sobre un aspecto determinado, se les pide que formulen ejemplos del tema tratado o que den referencias de autores en relación a la temática de la conversación.

Ejemplo:

"¿Cómo están? ¿Ya volvieron todos de unos días de descanso? ¿Listos para reanudar nuestro contacto?...espero que la respuesta sea "sí". Hasta el momento hemos recibido aportes de ...".

Ejemplo:

¿¿Se animan a compartir en este foro algunos ejemplos de textualizaciones didácticas? Esperamos sus intervenciones".

Ejemplo:

"...Quedo a la espera de sus comentarios y aportes que sin lugar a dudas van a enriquecer nuestro análisis en torno a los procesos de innovación y cambio educativo".

Ejemplo:

En tal sentido les consulto ¿qué aspectos del contexto pueden haber influido para que temas como los de Calidad y Equidad fueran incluidos a nivel de políticas? Quedo a la espera de sus aportes y les propongo avanzar el miércoles con otros aspectos, ¿les parece? 


\section{Reforzar una discusión agregando nuevos elementos de discusión}

Una forma interesante de intervención del docente apuntando a la profundización en la discusión del foro es la de traer a colación citas de autores, o la de solicitar aportes o reflexiones sobre la base de una lectura sugerida. De esta forma se genera una discusión académica con intercambios de calidad.

Ejemplo:

"Nos alienta ver que el número de las intervenciones va en aumento. En relación al planteo que hace Ana en el que destaca como principal objetivo de la educación el de "mejorar los aprendizajes", creo que agrega un elemento fundamental que nos permite entonces comprender mejor los conceptos de CALIDAD y de EQUIDAD... En relación al aporte que hace Cora y tomando en cuenta que uno de los autores que cita es Inés Aguerrondo, les propongo..."

Ejemplo:

"...Aguerrondo analiza diferentes aspectos del escenario institucional y de la gestión, que nos aportan herramientas de análisis para interpretar mejor la realidad de nuestros centros educativos, así como también algunas posibles estrategias de trabajo. Me gustaría mucho que Uds. me ayudaran a definir esas posibles herramientas y estrategias, teniendo en cuenta los aportes de Aguerrondo y los de otros autores que Uds. hayan leído y quieran incorporar.

Quedo a la espera de sus valiosos aportes".

\section{Generar socialización entre los alumnos del grupo virtual}

Los educadores a distancia pueden emplear diferentes técnicas para provocar socialización de los alumnos en el grupo virtual. Lograr esto es parte del éxito del foro, puesto que, si se logra involucrarlos entre sí, se pueden obtener intercambios más profundos y generar una discusión de mayor profundidad.

Ejemplo (interesante técnica que se va a describir más adelante):

"Estimados colegas: con singular agrado les doy una cordial bienvenida a este espacio de formación y actualización profesional. Por espacio de un mes tendré el placer de compartir con Uds. el desarrollo del Seminario "Concepciones de la enseñanza". En virtud de que vamos a conformar un grupo de trabajo, les propongo que durante estos primeros días nos vayamos conociendo. Para eso vamos a utilizar el correo electrónico. Les cuento algunas "pistas" de los integrantes de este grupo:

Hay dos colegas que en..."

\section{Motivar la participación de los alumnos}

Un modo de intervención muy frecuente de los docentes es el de reforzar la motivación de los alumnos que participan en el foro. Esto genera confianza en aquellos que aún no han participado y ven cómo son tenidos en cuenta los aportes individuales de los alumnos.

Ejemplo:

"Estimados: estoy muy contento con las intervenciones que están haciendo. Ya nos estamos acercando a entender los fenómenos del enseñar desde el propio campo de la enseñanza. María del Carmen, como buena alumna que es- lo tendré en cuenta para la calificación final-, realiza interesantes aportes reflexionando sobre..."

Ejemplo:

"Muy interesante este aporte de Raquel. Vean como nuestras 'configuraciones' desde la biografía personal y desde la socialización profesional, básicamente sobre el enseñar y las enseñanzas se impregna constantemente de un fuerte subjetivismo (e ideología). Pero no fue ése el discurso que emanaba de la "vieja didáctica", aquélla...."

Ejemplo:

"Estimados colegas: con singular agrado veo que están leyendo mucho sobre Didáctica. Los aportes que han realizado en este último día tanto Sara como Elena y Carolina son sustanciales. 
Es interesante observar cómo..."

Ejemplo:

"Estimados colegas: es increíble todo lo que hemos podido avanzar en estas semanas. Realmente sus propuestas y sus intervenciones impulsan a seguir trabajando más y más sobre el tema".

\section{Motivar la participación individual de un alumno}

Otra conducta observada en los docentes es la de entusiasmar y motivar a los alumnos rezagados cuando se integran a la discusión en el foro. Esto produce confianza y anima al alumno a continuar participando.

Ejemplo:

"Antonella; te damos la bienvenida y nos alegra que después de varios intentos, finalmente te encuentres ya integrada a esta red. Creo que tus aportes han complementado de forma excelente la temática en la que venimos trabajando.

Esperamos nuevas intervenciones".

\section{Resumir la discusión del foro como forma de provocar la participación de alumnos rezagados}

Una vez que ha transcurrido un tiempo prudencial desde el comienzo del foro, el docente, como recurso para provocar la participación de los alumnos rezagados, realiza un resumen de la discusión del foro. Para los alumnos que recién se integran, esta técnica resulta muy adecuada puesto que les permite comenzar a intervenir sin sentirse ajenos a la discusión que se venía desarrollando.

Ejemplo:

"Estimados colegas: espero que todos se encuentren bien y con ganas de avanzar en nuestra reflexión conjunta. Estuvimos intercambiando aportes y opiniones en torno a aspectos macroestructurales relacionados con las demandas del contexto y analizamos algunos objetivos de política educativa, temas que podrán profundizar en los textos..."

\section{Análisis de algunas de las técnicas empleadas para provocar intercambios interpersonales de calidad}

Técnica 1

Aquí presentamos un ejemplo de propuesta didáctica que genera intercambios de calidad. El docente "B" utiliza como recurso didáctico una técnica muy interesante con el objetivo de generar socialización, conocimiento personal e intercambios profundos. Solicita una reflexión desde la historia personal de cada uno:

PROFESOR "B": "Nuestro propósito es estudiar las prácticas de enseñanza. Es por eso que les propongo que cada uno de nosotros aporte, desde su historia personal, acerca de lo que cada uno de nosotros cree que es el Enseñar, acerca del oficio de enseñante... No buscamos acá, por el momento, definiciones de corrientes y/o autores, sino una primera conceptualización desde nuestra historia (historia como alumnos y como docentes). Los escucho. Yo también voy a intervenir... pero en un ratito para que no piensen que no los voy a dejar hablar.

Tienen la palabra...".

Es interesante ver cómo el docente está buscando generar aprendizajes significativos, puesto que primero hace reflexionar sobre los conocimientos previos, dejando de lado los autores, para luego incorporarlos al entramado conceptual.

ALUMNA 1: "...Me quedó en mente lo de responder sobre el oficio de enseñante desde la 
historia personal, y quise recordarme como alumna..."

ALUMNA 2. "...El interrogante me lleva a tiempos muy duros en los que me formé en la secundaria y en formación docente. Mi rol de aprendiz, lo recuerdo como un ser pasivo, sin mucha intervención desde la creatividad, donde la palabra del enseñante era palabra mayor, sin cuestionarme demasiado las supuestas verdades que se me impartían como tales..."

La observación de ambas participaciones denota que las dos alumnas se involucraron fuertemente con la propuesta y lograron un alto grado de apertura personal, lo que generó intercambios personales entre ambas:

ALUMNA 1 dirigiéndose a la ALUMNA 2: hola (nombre de la alumna 2), soy (nombre de la alumna. 1) Mirá estuve leyendo tus aportes. Es cierto, no cuestionábamos nada... Era una especie de status quo... "Estar del otro lado", del lado del enseñante te cambia: lo primero es un problema de perspectivas. El que está sentado, y el que está ahí mediando entre tantas cosas a las que seguramente iremos dando nombre durante el seminario con (nombre del docente).

Luego de varias intervenciones, el docente envía un mensaje electrónico conectando la discusión de las experiencias personales con las aportaciones del marco teórico de referencia:

"Todas sus intervenciones, estimados colegas, nos han recordado posibles vínculos entre los paradigmas dominantes en la institución educativa y nuestros procesos de formación. Me gustaría colocar en debate una afirmación que hace Davini, en el texto citado en la guía, al referirse a las tendencias no consolidadas las tradiciones. 'Más allá de estos intentos, las tendencias...'" ".

\section{Técnica 2}

Con la descripción de este recurso didáctico se ejemplifica cómo con acciones bien dirigidas, los docentes pueden producir un inicio dinámico del foro, rompiendo "el hielo" y las timideces iniciales, generando el sentimiento de pertenencia a un grupo y conocimiento personal.

El docente envía este mensaje:

"Estimados colegas: con singular agrado les doy una cordial bienvenida a este espacio de formación y actualización profesional.

Por espacio de un mes tendré el placer de compartir con Uds. el desarrollo del Seminario (nombre de la asignatura). En virtud de que vamos a conformar un grupo de trabajo, les propongo que durante estos primeros días nos vayamos conociendo. Para eso vamos a utilizar el correo electrónico. Les cuento algunas "pistas" de los integrantes de este grupo:

- Hay dos colegas que trabajan en la Escuela Agraria de Río Negro. -Otra colega anda enseñando filosofía por el este del país. - En Montevideo, me enteré de que uno de los participantes es profesor de Educación Física (pidámosle alguna sugerencia para estar en forma). -La más joven del grupo se llama Victoria. - Desde Mercedes asisten tres colegas (L, J, $\mathrm{SH}$ - son las iniciales de sus nombres). - Dicen que Zoraida habla muy bien el portugués ... y también Clara es psicológica no hay nada mejor que ir rumbo al este... bien al este, hasta llegar a la frontera. - Dos maestros nos acompañan desde el departamento "más chico" del país ¿quiénes serán? - Y también dos maestras de Montevideo, una se llama Nora, la otra se hace llamar María. - De la capital nacional al litoral norte. Dos salteñas: una es Prof. de Literatura, la otra Prof. de Biología y directora de liceo (las conozco muy bien a las dos tengo más datos por si ...). - Y volvemos a Montevideo, para decirles que tenemos en el grupo a una Prof. de Id. Español y psicopedagoga.

Tarea: intercambiar mensajes (por el correo electrónico, no por el foro) para saber más sobre nosotros.

Nos vemos en un ratito". 
Como resultado de esta propuesta, se produjeron intercambios personales y profundos entre los alumnos, generando un clima de gran entendimiento y de respeto en el grupo virtual. Los siguientes ejemplos ilustran el ánimo entre los participantes.

Ejemplo 1:

"Hola (nombre de alumna 3), soy (nombre de alumna 4). Leyendo tu vida escolar y los aportes que nos acercas, siento que tengo que felicitarte, creo que has dicho cosas importantes. Yo ingresé al ámbito escolar a los tres años de edad. Eso determinó en mí cierta natural indiferencia creo, cierta manera de vivir mi ser "alumna" como un devenir más. Entonces me entusiasmó mucho tu historia escolar vivida tan intensamente. ¡Gracias! Creo que eso me enriquece mucho a la hora de seguir valorando la presencia activa de mis aprendices de hoy".

Ejemplo 2:

"Gracias por tu testimonio (nombre alumna 5), creo que compartimos esa "pasión" de la que hablas: Enseñar... EDUCAR, iqué compromiso! Yo "enseño" literatura, estamos muy cerca en cuanto a disciplinas... Me alegro de que nos podamos comunicar, este espacio será fructífero".

\section{Conclusiones}

1. Existe una relación directa entre una intervención activa del docente que dirige el foro y una buena participación de los alumnos.

Una activa participación del docente en el foro condiciona positivamente la dinámica de las intervenciones de los alumnos. Esto surge claramente al comparar los indicadores del Foro del Seminario 2 del Profesor "B" en los años 2000 y 2001. La participación del docente aumentó al triple y el tiempo de respuesta del docente a las preguntas de los alumnos disminuyó a la tercera parte. Esto produjo un aumento significativo en el promedio de participación de los alumnos (aumentó casi al triple). Esta conclusión de que la participación activa del docente condiciona la actividad de los alumnos se afirma aún más si se observa la participación de la docente "A", que tuvo prácticamente el mismo promedio de intervenciones en los dos foros, y se reflejó en la actividad de los alumnos, que fue casi igual. El cambio que se produjo fue la leve disminución, en el segundo foro, del tiempo de respuesta a las demandas de los alumnos, provocando un pequeño aumento en el promedio de participación de los alumnos, pero poco significativo.

Por todo esto podemos afirmar que con un mayor aumento de participación del docente y con tiempos de demora de respuesta bajos, se motiva a los alumnos provocando un aumento en la participación de los alumnos en el foro.

2. Las acciones didácticas del docente son clave para dinamizar los foros de discusión de cursos que se desarrollan en la modalidad a distancia.

La utilización por parte del docente de distintas dinámicas grupales, como la técnica 2 anteriormente descripta, provoca un fluido intercambio de mensajes al comienzo del foro, y un conocimiento personal entre los integrantes del grupo, generando relaciones interpersonales más fuertes, lo que produce mayor integración grupal y compromiso con el curso y las tareas asignadas.

\section{La utilización de diferentes técnicas didácticas genera intercambios interpersonales profundos y de calidad.}

Una práctica activa y motivadora del docente, como la técnica 1 descripta anteriormente, repercute notoriamente en las participaciones de los alumnos en el foro, y puede generar intercambios de calidad, profundos e intensos. Las conversaciones se centran en aspectos relevantes, aportando al entramado conceptual, partiendo de los conocimientos previos, para dar paso a los nuevos conocimientos con los aportes de teoría, esto es, produciendo un aprendizaje significativo. 
4. Los alumnos de postgrado, docentes de profesión, en el Uruguay, se conectan mayoritariamente los días hábiles en el horario nocturno.

Para una buena práctica de enseñanza a distancia, el docente debe conocer el patrón de comunicación de su grupo, saber los días y horarios en que preferentemente se conectan los alumnos. Para grupos de características similares a los estudiados, docentes en la etapa de la formación de postgrado en Uruguay, se comunican mayoritariamente los días hábiles, de lunes a viernes, por la noche, y con poca frecuencia los fines de semana. Esto ocurrió en los cuatro foros estudiados. A partir de esta información es recomendable que el docente participe de acuerdo al patrón de comunicación de su grupo virtual, por lo menos, una vez al día los días de semana en el horario en que lo hacen habitualmente los alumnos.

5. Propuestas de intervención didáctica de los docentes en los foros estudiados.

El educador a distancia utiliza diferentes modos de intervención didáctica en los foros como forma de estimular y conducir las participaciones de los alumnos. Entre las categorías se destacan intervenciones para:

1.Iniciar una línea temática de discusión.

2.Encauzar una discusión.

3.Generar participación de los alumnos en el foro.

4.Reforzar una discusión agregando nuevos elementos.

5.Generar socialización entre los alumnos del grupo virtual.

6.Motivar la participación de los alumnos.

7.Motivar la participación individual de un alumno.

8.Resumir la discusión del foro como forma de provocar la participación de alumnos rezagados.

\section{Bibliografía y notas}

Brito, V. 2004. El foro electrónico: una herramienta tecnológica para facilitar el aprendizaje colaborativo. Revista Electrónica de Tecnología. No. 17. Edutec.

Bruno, E. 1999. Aprendizaje Colaborativo. Revista de Pedagogía No. 59.

Gallego, D. et al. 2000. Unidad didáctica 22: "Medios y recursos Tecnológicos en la Enseñanza a distancia". Manuales de la Maestría EAAD, UNED.

Gallego, M. 2003. Intervenciones formativas basadas en www para guiar el inicio de la práctica profesional de los docentes. Revista Iberoamericana de Educación. OEI No. 33 Setiembre-Diciembre 2003. www.campus-oei.org/revista/rie33a06.htm

García Aretio, L. 2000. Unidad didáctica 50: "Planificación de la enseñanza y aprendizaje abiertos y a distancia". Manuales de la Maestría EAAD, UNED.

García Aretio, L. 2000. Unidad didáctica 3: "Teoría y componentes de la Educación a Distancia". Manuales de la Maestría EAAD, UNED.

García Aretio, L. 2001. Educación a distancia. De la teoría a la práctica. Barcelona, Ariel.

Icaza, J. 2000. Estrategias didácticas en grupos de diálogo electrónico. http:// www.ruv.itesm.mx/estructura/dgacit/staff/manzana/articulos/articulo01.html

Mazzotti, W. y Laurta, E. 2000. Estudio sobre los factores que inciden en las interacciones interpersonales que tienen lugar en escenarios educativos no presenciales basados en interlintranet. Trabajo final, Diploma en Educación. Universidad ORT. 
Medina, A. 2000. Unidad didáctica 15: "El docente ante la enseñanza: papeles, tareas y acciones más representativas". Manuales de la Maestría EAAD, UNED.

Pallo y Pratt. 1999. http://prometeo.us.es/teleformación, citado en C. Marcelo et al (2002)

Restrepo J. 1973. "Lo humano en la comunicación social". Pontificia Universidad Javeriana. Bogotá en http://investigación.ilce.edu.mx/dice/diplomado/html/3html.html.

1 "La Educación en cuanto objeto de conocimiento e investigación es mucho más que esa realidad privilegiada y profunda del acto educativo, un acto en que se encuentran, al menos, dos personas en toda su complejidad: sus capacidades y aptitudes, sus sentimientos y afectos, sus actitudes, sus apreciaciones y valores ... y ello no tanto desde la realidad de las mismas sino desde la mutua percepción, siempre subjetiva" (Pérez Juste, R.).

${ }^{2}$ Morgan (1985) y otros han insistido en que la interacción necesaria en todo proceso educativo tiene que incentivar para que los alumnos sean activos, aumentar la comunicación entre los alumnos y docentes, y acrecentar la retroalimentación entre ellos.

3 "La calificación virtual de estos grupos se refiere a que en su conformación no tienen lugar las categorías de 'espacio' y 'distancia'. Los cibergrupos no ocupan, como tales, un lugar en el espacio y las distancias físicas entre sus miembros son irrelevantes" Roquet García.

"Las comunidades virtuales son definidas como espacios de intercambio comunicativo entre colectivos con un interés común" Gallego (2003).

${ }^{4}$ TIC: Tecnologías de la Información y la Comunicación.

${ }^{5}$ García Aretio define la educación a distancia como "un sistema tecnológico de comunicación bidireccional (multidireccional) que puede ser masivo, basado en la acción sistemática y conjunta de recursos didácticos y el apoyo de una organización y tutoría que, separados físicamente de los estudiantes, participan en éstos un aprendizaje independiente y cooperativo (García Arieto, 2001: 39).

6 "Los estilos de aprendizaje son los rasgos cognitivos, afectivos y fisiológicos, que sirven como indicadores relativamente estables, de cómo perciben los discentes, interaccionan y responden a sus ambientes de aprendizaje." (Gallego, 2000:234). Los estilos de aprendizaje en niveles de adultos desde la perspectiva de David Kolb son cuatro: Estilo Acomodador (competencias de "acción"), Estilo Divergente (competencias de "valorar"), Estilo Asimilador (competencias de "pensar") y Estilo Convergente (competencias de decisión"). Unidad didáctica 12: 238. Manuales de la Maestría EAAD, UNED.

${ }^{7}$ Citado por L. García Aretio (2000) en la Unidad Didáctica 50 "Planificación de la Enseñanza y Aprendizaje Abiertos y a Distancia", Manuales Maestría EAAD, UNED. 
${ }^{8}$ Se dice que el aprendizaje es significativo si el individuo realiza un esfuerzo deliberado y consigue relacionar la nueva información con conceptos ya adquiridos, incorporándose a la estructura cognitiva del alumno. Ausubel desarrolla una teoría del aprendizaje significativo en la que lo contrapone al aprendizaje memorístico. El aprendizaje significativo es aquel que el sujeto lleva a cabo de modo activo, estableciendo relaciones y conexiones entre las informaciones nuevas con las ideas previas que el sujeto tiene en su estructura mental. La estructura mental viene determinada por el modo en que los conceptos están organizados en la mente (Antonio Colmenar Santos).

${ }^{9}$ La Androgogía es la ciencia que estudia la forma en que aprenden los adultos. Establece que la forma de aprender de éstos difiere de la de los niños y jóvenes.

${ }^{10}$ Hoyle, recogidas por L. Stenhouse, citado por R. Pérez Juste (2000).

${ }^{11}$ Citado en Parker, 1997; en García, 2000.

${ }^{12}$ Cuando nos centramos en las estrategias de intervención estamos teniendo presente la opinión de Relan y Gillani, 1997, que define la formación mediante internet como "la aplicación de un repertorio de estrategias instruccionales orientadas cognitivamente y llevadas a cabo en un ambiente de aprendizaje constructivista y colaborativo, utilizando los atributos y recursos de Internet".

${ }^{13}$ La extensión de este artículo no permite presentar en detalle los resultados del análisis de los indicadores estudiados.

${ }^{14}$ Los nombres de los participantes fueron modificados para preservar sus identidades.

* Master en Educación abierta y a distancia, Universidad Nacional de Educación a Distancia (UNED), España. Diploma en Educación, Universidad ORT. Profesor de Física, Instituto de Profesores Artigas. Director, Enseñanza Secundaria, IUDEP. Docente e investigador, Instituto de Educación. 